\title{
Large-scale DNA nanoarrays with controllable fluorescence switch constructed by RCA simplified origami
}

\author{
Zhiqing Zhang, * Jie Ma, Guodong Zhang, * Xiaoyan Ding, Ruyan Zhang, Ting Zhou,
} Xiufeng Wang, and Fang Wang

Department of Chemistry, College of Science, China University of Petroleum (East China), Qingdao, 266580, P. R. China

*Email: zhangzq@upc.edu.cn, guodongzhang@upc.edu.cn

Table of Contents

Number of figures: 3

Number of tables: 1

Page S1: Table S1. DNA sequences used in DNA origami.

Page S2: Figure S1. Morphology of RCA-amplified ssDNA. (a) TEM image and (b) SEM image of ssDNA clustered structure.

Page S2: Figure S2. The agarose gel electrophoresis of RCA scaffolds.

Page S2: Figure S3. Agarose gel electrophoresis of assembly products at different molar ratio of

RCA scaffolds to staple strands.

Table S1. DNA sequences used in DNA origami. (Bold italic: sequence of thrombin aptamer)

\begin{tabular}{|c|c|c|}
\hline Name & & Sequence \\
\hline \multirow[t]{2}{*}{ circular template } & 1 & $\begin{array}{l}5^{\prime} \text {-TGTCTTCGCCTTCTTGTTTCCTTTCCTTGAAACTTCTTCCTTTCTT } \\
\text { TCTTTCGACTAAGCACC-3' }\end{array}$ \\
\hline & 2 & $\begin{array}{l}\text { 5'-TAAGATGAAGATAGCGCACAATGGTCGGATTCCGTCTCTGTCA } \\
\text { ACTCGTCTATGCCAAGCCCT-3' }\end{array}$ \\
\hline \multirow[t]{2}{*}{ RCA primer } & 1 & $\begin{array}{l}\text { 5'-GGCGAAGACAGGTGCTTAGTC-3' } \\
\end{array}$ \\
\hline & 2 & 5'-CTTCATCTTAAGGGCTTGGCA-3' \\
\hline \multirow[t]{6}{*}{ staple strands } & 1 & 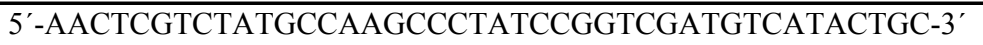 \\
\hline & 2 & 5'-TGGTCGGATTCCGTCTCTGTCCAATAGTCCGATACGTACCGT-3' \\
\hline & 3 & $\begin{array}{l}\text { 5'-TAAGATGAAGATAGCGCACAAATTAACGGGTAATCGACAGTC- } \\
\text { 3' }\end{array}$ \\
\hline & 4 & 5'-TTTCCTTGAAACTTCTTCCTTGACTGTCGATTACCCGTTAAT-3' \\
\hline & 5 & 5'-TCTTTCTTTCGACTAAGCACCGCAGTATGACATCGACCGGAT-3' \\
\hline & 6 (FAM-aptamer & 5'-FAM-GGTTGGTGTGGTTGGTTTTTGTCTTCGCCTTCTTGTTTCCA \\
\hline control DNA & 1 & 5'-FAM-GGTTGGTGTGGTTGG \\
\hline
\end{tabular}



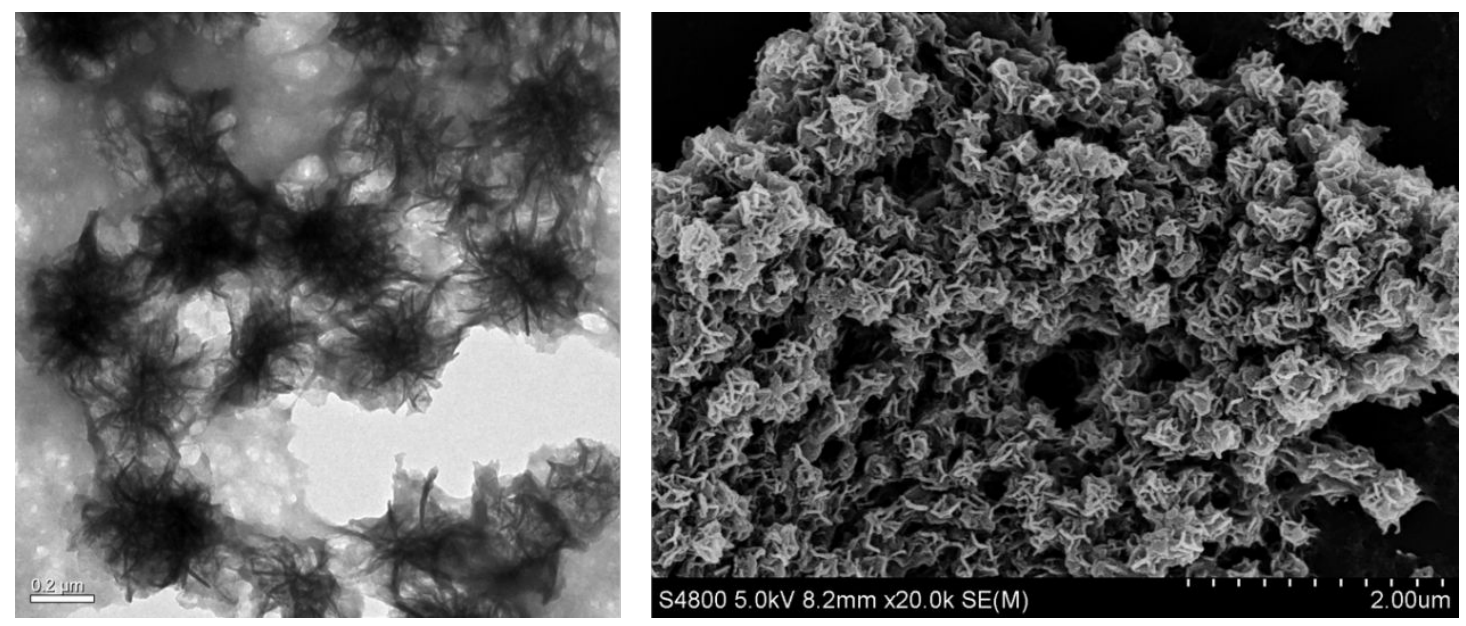

Figure S1. Morphology of RCA-amplified ssDNA. (a) TEM image and (b) SEM image of ssDNA clustered structure.

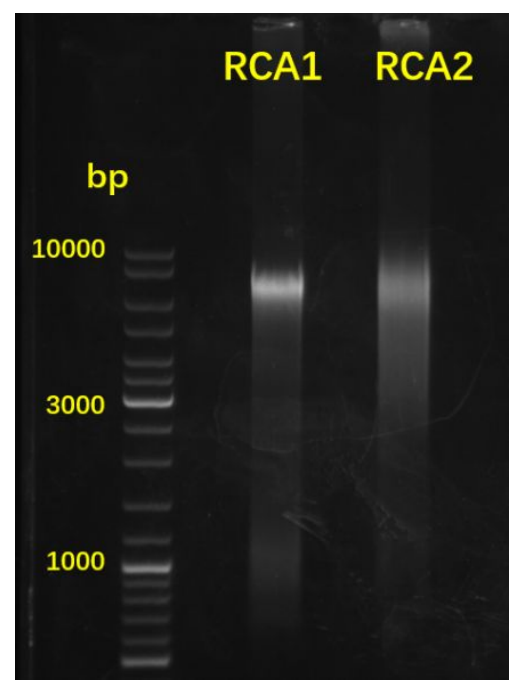

Figure S2. The agarose gel electrophoresis of RCA scaffolds.

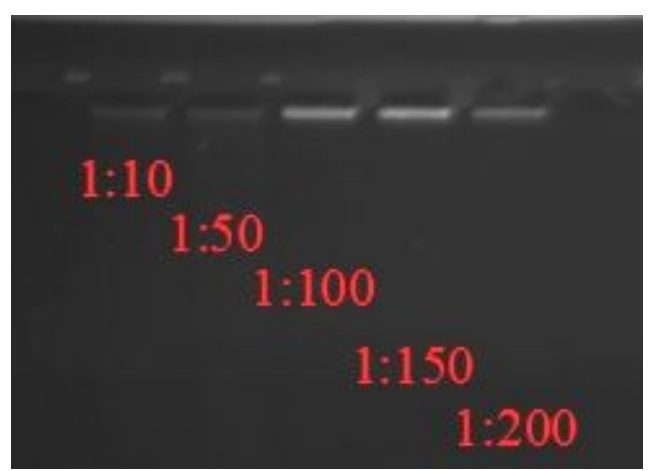

Figure S3. Agarose gel electrophoresis of assembly products at different molar ratio of RCA scaffolds to staple strands. 